not fulfill the criteria but were regarded as possible Sjögren's syndrome under development by the rheumatologist who investigated them.

Results: 196 of the 199 pSS patients also fulfilled the new criteria from 2016. The three patients who did not only had SSB autoantibodies and no positive biopsy. Of eight patients with suspicion of Sjögren's syndrome under development, three fulfilled the new criteria.

Conclusions: The classification criteria for Sjögren's syndrome from 2002 and 2016 are consistent. This study indicates a possibility that the new criteria may be more sensitive early in the disease development, as long as the classification is not based on autoantibody positivity against SSB.

Disclosure of Interest: None declared

DOI: 10.1136/annrheumdis-2017-eular.5377

\section{THU0281 LONG-TERM PROGNOSIS AND PREDICTINGFACTORS OF CHINESE PATIENTS WITH SYSTEMIC LUPUS ERYTHEMATOSUS: A MULTI-CENTER COHORT STUDY FROM CSTAR REGISTRY}

M. $\mathrm{Li}^{1}$, Z. Wang ${ }^{1}$, Y. Wang ${ }^{2}, \mathrm{X}$. Zeng ${ }^{1}$ on behalf of CSTAR. ${ }^{1}$ Peking Union Medical College Hospital; ${ }^{2}$ Chinese Academy of Medical Science, Beijing, China

Objectives: To investigate the long-term outcomes, both mortality and damage, and related prognostic factors of patients with systemic lupus erythematosus (SLE) in the CSTAR (Chinese SLE Treatment and Research group) registry cohort.

Methods: All of the patients were enrolled from April 2009 to February 2010. They were followed up at clinic and were telephone interviewed at the endpoint. Demographic data, clinical manifestations, activity (SLEDAI-2K), damage scores (SLLIC/Damage Index), and medications were collected. Data were censored at the last clinic visit or telephone interview. Survival rates were studied by Kaplan-Meier method, and COX proportional hazard model was adopted to perform the analysis of predicting factors for mortality.

Results: A total of 2104 patients were recruited at baseline, and 1494 patients were successfully followed up. The cumulative 1, 3 and 5-year survival ratesfrom diagnosis were $99.0 \%, 98.1 \%$ and $97.1 \%$. 78 patients died during follow-up, and the main death causes were infection (34.6\%), active disease (26.9\%), cardiovascular and cerebrovascular events $(6.41 \%)$ and malignancy $(5.13 \%)$. At entry, 247 patients presented with irreversible organ damage and it increased to 398 patients at the endpoint. The major accumulated organ damages were renal $(25.9 \%)$, musculoskeletal $(20.2 \%)$, neuropsychiatric $(12.4 \%)$, and pulmonary $(10.8 \%)$ damage. Cox regressionshowed that male, late onset age ( $>50 \mathrm{y})$, onset to diagnosis time $\geq 1$ year, previous organ damage, renal involvement, pulmonary arterial hypertension, neuropsychiatric involvement, serositis and the number of involved organ systems $\geq 3$ predict for higher mortality.

\begin{tabular}{llll}
\multicolumn{4}{l}{ Table 1: Independent predictors of mortality obtained by univariate analysis } \\
\hline Univariate analysis & $\mathrm{HR}$ & $95 \% \mathrm{Cl}$ & $p$ \\
\hline Age at onset $\geq 50$ years & 3.935 & $2.111-7.334$ & $<0.001$ \\
Onset to diagnosis $\geq 1$ year & 1.996 & $1.251-3.186$ & 0.004 \\
Gender & 2.082 & $1.119-3.873$ & 0.021 \\
Baseline organ damage* & 2.846 & $1.757-4.610$ & $<0.001$ \\
Renal involvement* & 2.434 & $1.425-4.156$ & 0.001 \\
Hematologic involvement* & 1.605 & $0.978-2.635$ & 0.061 \\
Interstitial lung disease* & 2.167 & $0.973-4.823$ & 0.058 \\
Pulmonary arterial hypertension* & 4.126 & $2.107-8.081$ & $<0.001$ \\
Neuropsychiatric involvement* & 2.290 & $1.169-4.486$ & 0.016 \\
Serositis* & 2.580 & $1.605-4.148$ & $<0.001$ \\
No. of involved organ systems (1) * & 1.793 & $0.719-4.466$ & 0.210 \\
No. of involved organ systems (2) * & 2.045 & $0.805-5.195$ & 0.133 \\
No. of involved organ systems ( $\geq 3)^{*}$ & 5.638 & $2.339-13.589$ & $<0.001$ \\
\hline
\end{tabular}

* Performed after controlling of gender, onset age and time from onset to diagnosis

Conclusions: Long-term survival rates have improved for Chinese SLE patients. Early diagnosis, preventing fromthe emerging systemic organ involvements and organ damagecould be the treating target for the management of SLE patients in China.

Disclosure of Interest: None declared

DOI: 10.1136/annrheumdis-2017-eular.5791

\section{THU0282 HOMOCYSTEINE: ANY ROLE IN PERIPHERAL VASCULAR DISEASE IN SYSTEMIC LUPUS ERYTHEMATOSUS (SLE) PATIENTS?}

N. Mohannad $^{1}$, M. Tayel ${ }^{2}$, M.H. Megallaa ${ }^{3} .{ }^{1}$ Internal Medicine, Rheumatology Unit, Alexandria University Hospitals, Alexandria University; ${ }^{2}$ Internal Medicine, Rheumatology Unit; ${ }^{3}$ Internal Medicine, Alexandria University, Alexandria, Egypt

Background: Many are the independent risk factors for premature atherosclerosis in general \& peripheral vascular disease (PVD) in particular in SLE patients. Plasma homocysteine (HCY) is a known risk factor for atherosclerosis. Atherosclerosis can lead to many cardiovascular diseases as myocardial infarction, stroke and claudication
Objectives: To compare the occurrence PVD of the lower extremity of SLE patients (pts) with age and sex matched controls and evaluate the role of HCY level in its occurrence

Methods: Body mass index (BMI), blood pressure, lipid profile, titers of autoantibodies [ANA, anticardiolipin antibodies ACL (IgM, IgG)], C3, C4, plasma HCY level were assessed, SLEDAI and (SLICC/ACRDI)were calculated. PVD evaluation was done by measuring Ankle Brachial Index (ABI) with values $<0.9$ considered diagnostic of PVD; in 60 SLE pts and 30 age-matched controls. Patients with previous hypertension, diabetes, other collagenic diseases \& smokers were excluded

Results: Eighty-eight percent of the pts were women. The mean age (SD) was 30.40 (11.46) years \& mean disease duration 3.61 (4.92)years. 50 pts were asymptomatic, 5 had mild \& 5 had moderate claudications. SLE pts had significant higher total cholesterol (TC), LDL than controls $224.1 \pm 57.8$ vs $181.1 \pm 41.1 \mathrm{mg} / \mathrm{dl}$ \& $162.7 \pm 57.0$ vs $119.5 \pm 13.4\left(<0.001^{*}\right)$ respectively, higher $\mathrm{HCY} 11.6 \pm 2.1$ \& $6.4 \pm 0.1 \mu \mathrm{mol} / \mathrm{L}\left(\mathrm{p}<0.001^{\star}\right)$ \& lower HDL $47.2 \pm 13.1 \& 52.6 \pm 3.7 \mathrm{mg} / \mathrm{dl}\left(\mathrm{p}=0.004^{\star}\right)$. Low $\mathrm{ABI}$ was found in $30 \%$ of SLE pts but none of the controls $\left(p=0.001^{*}\right)$ \& was correlated with higher HCY level $\left(p=0.005^{\star}\right)$, TC \& LDL $\left(p<0.001^{*}\right.$ \& $\left.p<0.001^{*}\right)$, but not TG $(p=0.748)$ or asymptomatic $p t s$, presence of mild or moderate claudications $(p=0.468,1.000,0.154)$, still its value negatively correlated with lupus anticoagulant (LA) $(p=0.002), A C L \operatorname{lgM}\left(p<0.001^{*}\right)$, the presence of lupus nephritis (LN) $\left(p<0.001^{*}\right)$ \& SLICC/ACRDI $\left(p=0.017^{*}\right)$ but not with disease duration (dd) $(p=0.535)$, Anti ds DNA ( $p=0.364)$, ACL IgG $(p=0.8940)$, C4 $(p=0.168)$ or SLEDAI $(p=0.074)$. No correlation was found between HCY level and pts' age, dd, age at diagnosis, BMI, Anti ds DNA, ACL IgG, C4 \& SLEDAI $(p=0.521,0.098,0.946,0.502,0.346,0.335,0.325,0.787)$. A positive correlation was found between HCY level and LA, ACL lgM, TC, LDL, the presence of LN \& SLICC/ACRDI with a p value of $0.025^{*},<0.001^{*},<0.001^{*}, 0.003^{*}, 0.001^{*}, 0.001^{*}$ \& negative one with $\mathrm{HDL} \mathrm{p}=0.023^{*}$
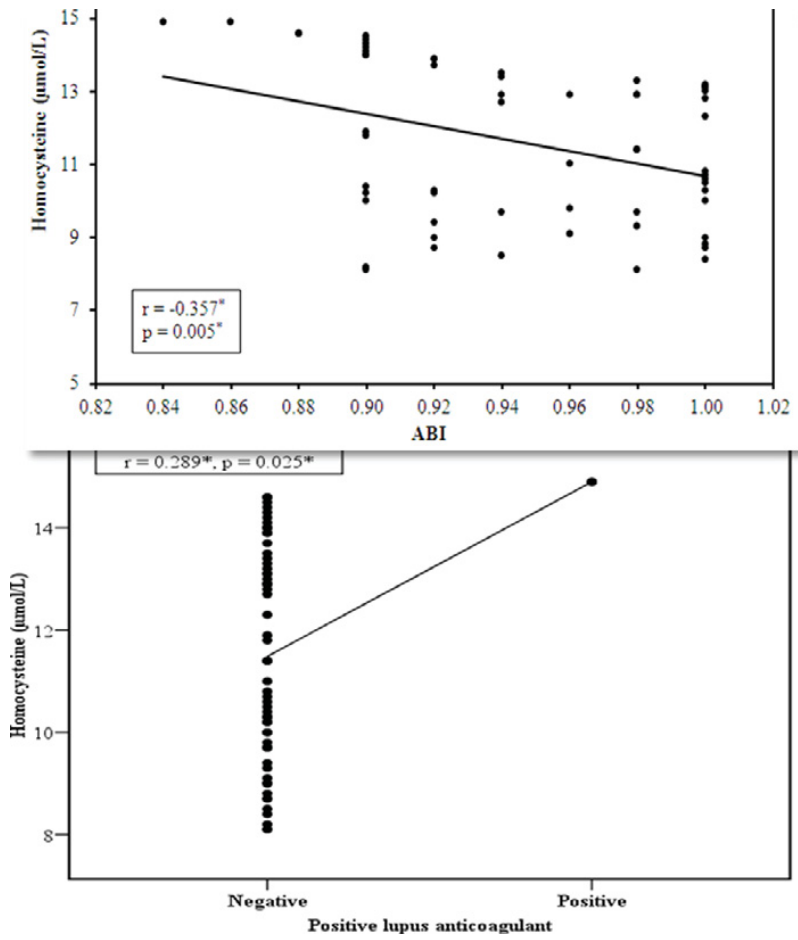

Conclusions: $83.3 \%$ of SLE pts were asymptomatic or had atypical symptoms of PVD still $30 \%$ of the patients had low $A B I$. ABI can be a more reliable, non-invasive test to assess PVD than the conventional methods of pulse palpation or history of claudication in SLE pts.Both traditional \& nontraditional risk factors of atherosclerosis are important but $\mathrm{HCY}$ can play a role, among other factors, as independent risk factor of PVD in SLE

References:

[1] Rayford R et al. PVD in SLE Patients. J Clin Rheum. 2013; 7:367-70.

Disclosure of Interest: None declared

DOI: 10.1136/annrheumdis-2017-eular.4615 